\title{
Oskar protein interaction with Vasa represents an essential step in polar granule assembly
}

\author{
Wolfgang Breitwieser, ${ }^{1}$ Finn-Hugo Markussen, ${ }^{1}$ Heinz Horstmann, ${ }^{2}$ and Anne Ephrussi ${ }^{1,3}$ \\ ${ }^{1}$ Developmental Biology Programme and ${ }^{2}$ Cell Biology Programme, European Molecular Biology Laboratory, \\ 69117 Heidelberg, Germany
}

\begin{abstract}
The posterior pole plasm of the Drosophila egg contains the determinants of abdominal and germ-cell fates of the embryo. Pole plasm assembly is induced by oskar RNA localized to the posterior pole of the oocyte. Genetics has revealed three additional genes, staufen, vasa, and tudor, that are also essential for pole plasm formation. Staufen protein is required for both oskar RNA localization and translation. Vasa and Tudor are localized dependent on Oskar protein and are required to accumulate Oskar protein stably at the posterior pole. We have explored interactions between these gene products at the molecular level and find that Oskar interacts directly with Vasa and Staufen, in a yeast two-hybrid assay. These interactions also occur in vitro and are affected by mutations in Oskar that abolish pole plasm formation in vivo. Finally, we show that in the pole plasm, Oskar protein, like Vasa and Tudor, is a component of polar granules, the germ-line-specific RNP structures. These results suggest that the Oskar-Vasa interaction constitutes an initial step in polar granule assembly. In addition, we discuss the possible biological role of the Oskar-Staufen interaction.
\end{abstract}

[Key Words; Drosophila; oogenesis; pole plasm; Oskar; Vasa; Staufen]

Received May 22, 1996; revised version accepted July 15, 1996.

In a wide variety of species, specific macromolecular structures have been identified that are located in the germ plasm, the region of the egg that gives rise to the germ-cell lineage. During development, the germ granules segregate specifically with the precursors of the germ cells (for review, see Eddy 1975; Lehmann and Rongo 1993). In Xenopus laevis, the germ plasm is localized to the vegetal cortex of the oocyte (Heasman et al. 1984|, and at blastula stage, is found incorporated in a number of presumptive primordial germ cells located within the endodermal mass (Whitington and Dixon 1975). In Caenorhabditis elegans, cytoplasmic structures, the P granules, localize to the posterior of the onecell embryo (Strome and Wood 1982,1983). Through a series of asymmetric divisions, the $\mathrm{P}$ granules become confined to the cells of the P lineage that leads to the germ-line fate (Strome and Wood 1982). In Drosophila melanogaster, pole plasm forms during oogenesis at the posterior pole of the oocyte as a specialized yolk-free cytoplasm containing electron-dense particles termed polar granules (for review, see St Johnston and Nüsslein-Volhard 1992). At stage 4 of embryogenesis, the polar granules are incorporated into the pole cells, the primordial germ cells. Only in Drosophila has the inductive capacity of germ plasm been demonstrated. Indeed, transplantation experiments have shown that pole plasm

${ }^{3}$ Corresponding author. can induce formation of germ cells at the site of injection (Illmensee and Mahowald 1974).

The Drosophila pole plasm is required not only for germ-line formation but also for development of the abdomen (Frohnhöfer et al. 1986). Based on the loss-of-abdomen phenotype as well as the absence-of-germ-line phenotype, a number of maternal genes have been identified that are involved in pole plasm formation (for review, see Lchmann 1995). The proteins of two of these gencs, vasa (vas) and tudor (tud), are localized at the posterior of the oocyte and have been shown to be components of the polar granules (Hay et al, 1988a,b; Lasko and Ashburner 1988; Bardsley et al. 1993). The localization of Vas and Tud proteins requires the activity of two other genes, oskar losk) and staufen (stau; Hay et al. 1990; Lasko and Ashburner 1990; Bardsley et al. 1993).

During oogenesis, a stem cell divides four times with incomplete cytokinesis, producing a syncytium that differentiates into the oocyte and 15 nurse cells. Oogenesis has been divided into 14 stages during which the oocytenurse-cell cluster undergoes morphological changes, resulting in the formation of the mature egg (King 1970). RNAs and proteins required for oocyte development are produced in the nurse cells and transported into the oocyte through cytoplasmic bridges formed by the ring canals. As early as stage 2, osk mRNA is found enriched specifically in the oocyte, and from stage 8 onward is concentrated at the posterior pole (Ephrussi et al. 1991; Kim-Ha et al. 1991). Shortly thereafter, Osk protein is 
detected at the posterior pole. Localization as well as translation of osk requires the double-stranded RNAbinding protein Stau, which colocalizes with osk mRNA during most stages of oogenesis (Ephrussi et al. 1991; Kim-Ha et al. 1991, 1995; St Johnston et al. 1991, 1992). Mislocalization of osk RNA to the anterior of the oocyte by expression of an osk-bcd3'UTR transgene results in the presence of Osk protein at the anterior, and subsequently in the formation of an ectopic pole plasm at the anterior of the embryo (Ephrussi and Lehmann 1992). This Osk-induced pole plasm does not require the activity of stau and other genes involved in the normal osk RNA transport to the posterior. However, vas and tud are required, revealing that these genes produce essential components of the pole plasm (Ephrussi and Lehmann 1992).

Translational control of osk mRNA generates two proteins, the Long Osk and Short Osk isoforms, consisting of amino acids 1-606 and 139-606, respectively, relative to the first methionine encoded by the cDNA (Markussen et al. 1995; Rongo et al. 1995). In the embryo, the two Osk species show distinct localization patterns. Long Osk is detected only until pole cell formation, whereas Short Osk persists and is incorporated into the pole cells. Expression of Short Osk alone is sufficient to rescue the defects of osk pole plasm mutants, whereas Long Osk alone shows no rescue activity (Markussen et al. 1995).

To understand the process of pole plasm assembly, we have studied the interactions of Osk with other known pole plasm proteins. Here we demonstrate that wild-type Osk protein interacts with Vas in the yeast two-hybrid assay and in vitro. Further, we show that mutations in Osk that abolish pole plasm formation also compromise the Osk-Vas interaction in vitro. These results suggest that the Osk-Vas protein interaction is an important step in anchoring Vas to the posterior pole of the oocyte, and a prerequisite for polar granule formation. We also find that Osk interacts specifically with Stau protein. Since Osk is required for maintenance of its own mRNA as well as of Stau at the posterior cortex (Ephrussi et al. 1991; St Johnston et al. 1991; Rongo et al. 1995), formation of an Osk-Stau protein complex could contribute to the stabilization function of Osk. Finally, we show that in stage 10 oocytes, Osk and Stau localize to the same regions at the posterior of the oocyte as Green Fluorescent Protein-Vas (GFP-Vas), a fusion protein that has vas activity and serves as a marker of pole plasm. In early embryos, Osk and GFP-Vas continue to colocalize at the posterior pole. Immunoelectron microscopy of embryos reveals that Osk, like Vas, is a component of the polar granules. Together with the fact that Osk recruits Vas and Tud to form the pole plasm (Ephrussi and Lehmann 1992), these results suggest that Osk nucleates polar granule assembly by a direct interaction with Vas.

\section{Results}

Osk interacts with Vas and Stau in the yeast two-hybrid assay

To test whether Osk protein undergoes direct interac- tions with other pole plasm proteins, we used a yeast interaction trap (Gyuris et al. 1993). We coexpressed the Short Osk isoform (residues 139-606), fused to the DNAbinding protein LexA (LexA-Osk), with various proteins fused to the transcription trans-activation domain B42, and tested their ability to activate expression of a $1 \mathrm{acZ}$ reporter gene bearing LexA-binding sites in its promoter (Fig. 1A,B). On X-Gal plates, yeast cells expressing LexAOsk show no lacZ activation in the presence of the activator alone. When $V$ as is fused to the activator, yeast cells display strong lac $Z$ activity. This activity is increased when only the amino-terminal half of Vas (residues $1-320$ ) is expressed. Little or no activity is detected when the carboxyl terminus alone (residues 446-661) is present (Fig. 1A). When LexA-Osk is expressed together with the amino-terminal half of Stau protein (residues 1-568) fused to the activator, strong lacZ activity is observed, whereas little or no activity is detected when a

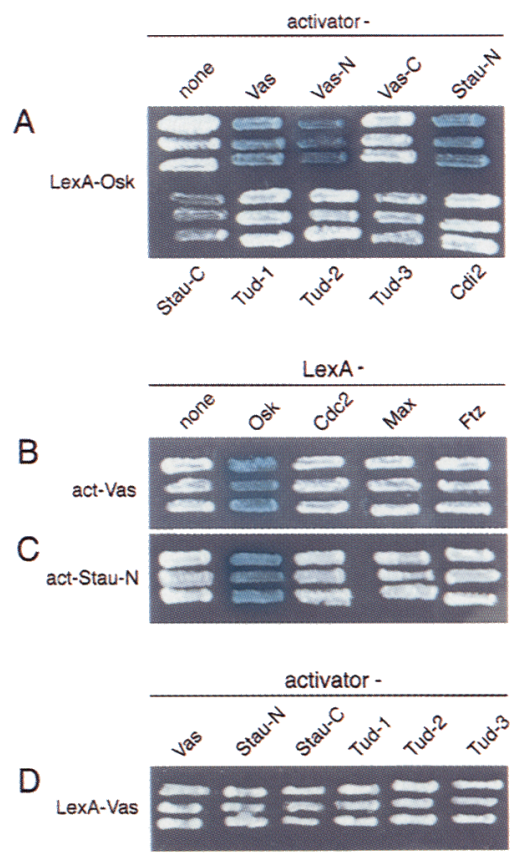

Figure 1. Two-hybrid assay for interactions between pole plasm proteins. $(A)$ LexA was fused to Short Osk (LexA-Osk), activator was fused to full-length Vas (Vas), Vas residues 1-320 (Vas-N), Vas 446-661 (Vas-C), Stau 1-568 (Stau-N), Stau 5691026 (Stau-C), Tud 1-271 (Tud-1), Tud 197-1197 (Tud-2), Tud 1198-2515 (Tud-3), and DmCdi2 (Cdi2). Each potential interaction was tested in three independent yeast clones. $\beta$-galactosidase activity is observed only in cells coexpressing Osk with Vas, Vas-N, and Stau-N. Note a stronger staining is observed in the presence of Vas- $\mathrm{N}$ than of full-length Vas. $(B)$ Activator-Vas and $(C)$ activator-Stau- $\mathrm{N}$ were tested for interaction with LexA alone, and LexA fused to Short Osk (Osk), DmCdc2 (Cdc2), Max, and the Ftz homeodomain $(\mathrm{Ftz})$. $\beta$-Galactosidase activity is only observed in the presence of Short Osk, confirming the specificity of the interaction. $(D)$ No activation is observed when LexA-Vas is tested for interaction with activator fused to Vas, Stau-N, Stau-C, and 3 Tud fragments. 
carboxy-terminal Stau peptide (residues 569-1026) is expressed (Fig. 1A).

Three portions of Tud protein, encoding amino acids 1-271, 197-1197, and 1198-2515, together comprising the complete Tud protein, were expressed as activatorfusions. Although the proteins were expressed abundantly (data not shown), no interaction was observed with LexA-Osk (Fig. 1A). Furthermore, LexA-Osk fails to interact with proteins that have no relation to pole plasm, including the Drosophila Cdc2 homolog (DmCdc2)-interacting protein Cdi2 (Fig. 1A) and the human Max-interacting protein Mxil (not shown). This further excludes the possibility that Osk binds proteins unspecifically in yeast. To show that Vas and the Stau amino terminus are specific in their interactions with Osk, we tested the ability of these proteins to interact with several different LexA-fusion proteins (Fig. 1B,C). Whereas Vas and Stau showed interaction with LexA-Osk, they failed to show any interaction with LexA alone, and LexA fused to DmCdc2, to Max, or to the Fushi-tarazu (Ftz) homeo domain. This confirms that, in yeast, Vas and the amino-terminal half of Stau engage in specific interactions with Osk.

To test whether Vas undergoes interactions with other pole plasm components in addition to Osk, LexA-Vas fusions were tested for $l a c Z$ activation in the presence of Stau- $\mathrm{N}$ and Stau-C peptides, the three Tud peptides, and Vas itself. In no case were blue-staining cells observed within two to three days of incubation at $30^{\circ} \mathrm{C}$ (Fig. 1D). However, after several days of incubation, yeast cells expressing both LexA-Vas and activator-Vas showed some activation of lacZ, suggesting the possibility of a weak homodimeric interaction of Vas. Taken together, of the interactions tested, we find that only Osk and Vas, and Osk and Stau, appear to interact directly and that the interaction domains are located in the amino-terminal halves of Vas and Stau, respectively. We cannot rule out, of course, that some interactions were missed in this assay, as interaction domains in Stau or Tud may have been split when the proteins were expressed as nonoverlapping activator-fusion peptides.

\section{Different abilities of Long Osk and Short Osk to interact with Vas and Stau}

Of the two Osk isoforms, Short Osk rescues the defects of osk pole plasm matants, whereas Long Osk shows no rescue activity (Markussen et al. 1995). We were interested therefore to know whether the two isoforms interact differently with Vas and Stau. To ascertain that a quantitative comparison between Long Osk and Short Osk could be made in our assay, we verified using a transcription interference assay (Brent and Ptashne 1984) that the two Osk isoforms bind to the LexA binding sites with similar efficiency. Normalizing to lac $Z$ activation by LexA alone, we find that LexA-Short Osk induces activity on average 13-fold when coexpressed with activator-Vas and 17-fold when coexpressed with activator-
Stau amino-terminus (Fig. 2). In contrast, LexA-Long Osk induces lac $Z$ activity more weakly, only three-fold in the presence of Vas and four-fold in the presence of the Stau amino-terminus (Fig. 2). This suggests that whereas both Long Osk and Short Osk can interact with Vas and Stau, only Short Osk does so efficiently.

To define further the regions in Osk that engage in interactions with Vas or Stau, we tested different portions of Osk (Fig. 2). As described above, we measured similar DNA-binding cfficiencies between LexA fusions to these truncated Osk proteins in the transcription interference assay. A carboxy-terminal fragment of Osk (residues 290-606) induces lac $Z$ activity 23 -fold in the presence of Vas, and 27-fold in the presence of Stau, demonstrating that at least one interacting domain of Osk lies within the carboxy-terminal region of the protein. Interestingly, this portion of Osk is more active in its interaction with Vas and Stau than entire Short Osk. A fragment of Long Osk bearing a carboxy-terminal deletion (residues 7-468) fails completely to interact with Vas and Stau. Similarly, when a fragment of the Osk carboxyl terminus alone (residues 451-606) is tested, no induction is observed. These results suggest that sequences between amino acids 451 and 606 are necessary, but not sufficient for interactions of Osk with Vas and Stau.

\section{Analysis of pole plasm interactions using bacterially expressed Osk}

To substantiate the results we observed in the yeast twohybrid assay, we wanted to reproduce the Vas and Stau interactions in vitro using purified Osk protein (Fig. 3). Because full-length Short Osk was unstable when ex-

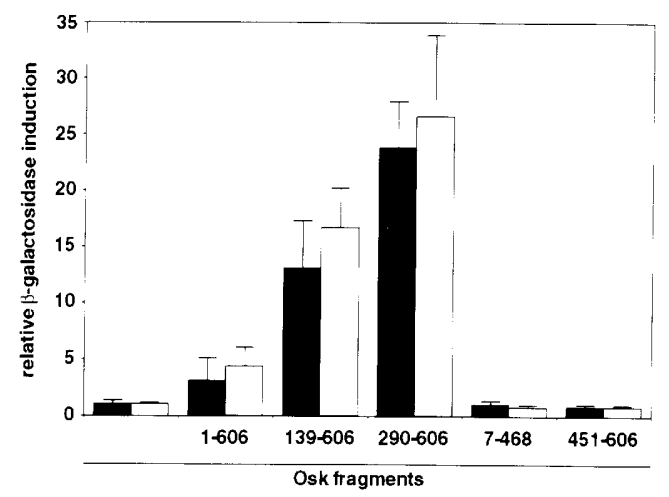

Figure 2. Interactions of Osk fragments with Vas (solid bar) or Stau residues 1-568 (Stau-N) (open bar). Inductions of $\beta$-galactosidase activity by LexA-Osk fragments are relative to induction by LexA alone. Strong activity is induced by Short Osk (139-606) or Osk(290-606), only weak activity is induced by Long Osk (1-606). No activity is induced by Osk(7-468) or Osk $|451-606|$. Osk residues $1-6$ are not necessary for interactions because an Osk fragment containing residues 7-606 and entire Long Osk (1-606) show very similar $\beta$-galactosidase activation with Vas or Stau-N (not shown). 


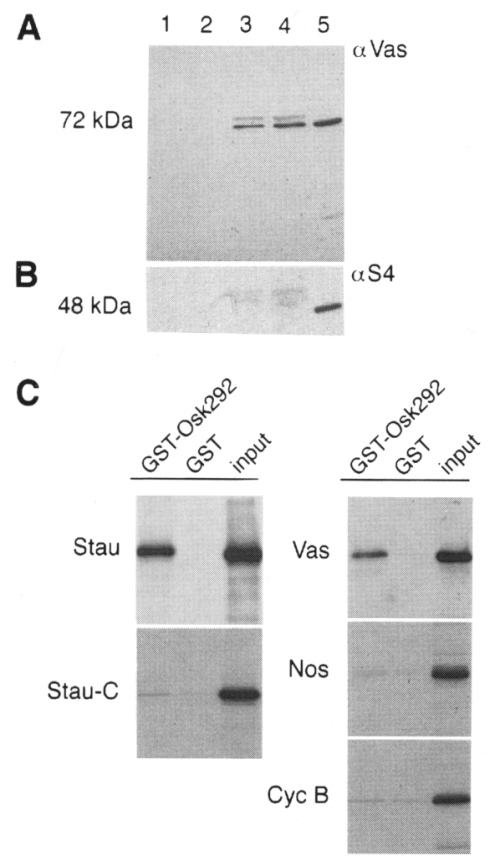

Figure 3. $(A, B)$ Vas from ovary extracts interacts specifically with GST-Osk292. (A) Polyclonal anti-Vas antibodies detect Vas in crude extracts (lane 5) and in samples incubated with 5 $\mu \mathrm{g}$ (3) or $10 \mu \mathrm{g}$ (4) of GST-Osk292 but not in samples incubated with $5 \mu \mathrm{g}(1)$ or $10 \mu \mathrm{g}$ (2) of GST alone. The anti-Vas antibodies cross-react with a protein of slightly larger size than Vas in samples containing GST-Osk292 $(3,4)$. This protein is not detected in ovary extracts but in samples containing purified GST-Osk292 (not shown), and is therefore a contaminant in the GST-Osk292 preparation. (B) Antibodies against the Drosophila homolog of the $26 \mathrm{~S}$ protease S4-subunit detect a specific band in ovary extracts but not in samples containing GSTOsk292 or GST. Note a weak cross-reaction of these antibodies against the very abundant GST-Osk292 $(3,4)$. (C) Stau and Vas made in vitro interact with Osk. Typically, $8 \%-10 \%$ of radiolabeled full-length Stau (Stau) and 1\%-4\% of full-length Vas (Vas) are retained by GST-Osk292, but insignificant amounts are retained by GST Stau residues 569-1026 (Stau-C), Nanos (Nos), and Cyclin B (Cyc B) are retained poorly by GST-Osk 292 and GST. In vitro-made Stau residues $1-569$ are retained strongly by GST-Osk292 but also, to a certain extent, by GST (not shown).

pressed in bacteria and could not be purified efficiently, a carboxy-terminal portion of Osk (residues 292-606) was expressed as a glutathione $S$-transferase fusion (GST-Osk292). The fusion protein was purified and immobilized on glutathione beads, and incubated with Drosophila ovary extracts. After extensive washing, bound proteins were released from the beads by boiling and separated by SDS-PAGE. Western blot analysis using Vas-specific antibodies reveals that Vas is detected in samples eluted from GST-Osk292, but not from samples that contain GST alone (Fig. 3A). To see whether this interaction is specific, we probed the same Western blot with antibodies against the Drosophila homolog of the proteasome S4 subunit, another abundant ovarian pro- tein (Fig. 3B; W. Breitwieser, unpubl.). A band is detected in samples containing crude extract, but not in samples eluted from GST or GST-Osk292.

With standard homogenization techniques it was not possible to obtain Stau protein in a soluble form from ovary extracts. Therefore, we tested the ability of GSTOsk292 to interact with in vitro-translated proteins (Fig. 3C). When ${ }^{35} \mathrm{~S}$-labeled Vas made in reticulocyte lysate is incubated with GST-Osk292 or GST, Vas shows specificity for GST-Osk292 but not for GST, although only a small percentage of the input protein is retained on the GST-Osk 292 beads. Stau produced in vitro is bound efficiently by GST-Osk292, but insignificantly by GST alone. As was observed in the two-hybrid assay, a carboxy-terminal portion of Stau (residues 569-1026) made in vitro is retained only weakly by GST-Osk292 and GST. Control proteins, including Nanos and Cyclin B, fail to interact with either GST-Osk292 or GST (Fig. $3 \mathrm{Cl}$.

Mutant Osk proteins are defective in their interactions with Vas and Stau

In all charactarized osk mutants, Vas protein fails to localize to the posterior pole of the oocyte. In these mutants, small deletions or point mutations result either in premature stop codons or in single codon changes in the osk coding sequence. It is remarkable that all eleven sequenced osk missense alleles contain mutations in the carboxy-terminal half of the protein, between positions 360 and 595. Because this region lies within the portion of Osk that interacts with Vas and Stau, we asked whether some of these mutations, when introduced into GST-Osk292, would affect interactions with these proteins in vitro (Fig. 4).

The Ser $457 \rightarrow$-Phe substitution, present in the allele

A
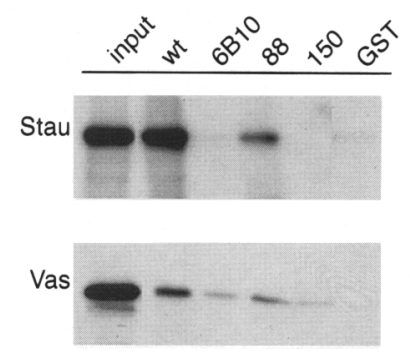

B

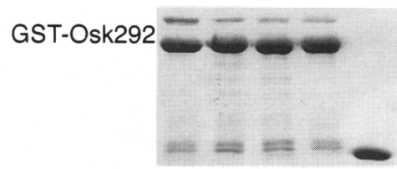

GST

Figure 4. Vas and Stau interactions are affected by mutations in Osk. (A) Compared to wild-type GST-Osk292 (wt), two mutant proteins $(6 \mathrm{~B} 10,150)$ are strongly defective in interactions, one $(88)$ is moderately defective. $(B)$ Coomassie-staining reveals that the same amounts of wild-type and mutant GST-Osk292 proteins as well as GST protein alone were used. 
${ }^{o s k}{ }^{6 B 10}$ (C. Rongo and R. Lehmann, pers. comm.), causes a severe reduction in both the Osk-Vas and the OskStau interactions (Fig. 4A). The Asn561 $\rightarrow$-Tyr substitution, corresponding to the allele os $k^{88}$, results in a weaker, but still significant decrease in interaction with Vas as well as with Stau (Fig. 4A). The Arg 593 $\rightarrow$-Gln substitution of the allele $\operatorname{osk}^{150}$ causes a dramatic decrease in the interactions with Vas and Stau (Fig. 4A). Hence, all three osk missense mutations cause a significant reduction in the efficiency of interaction of Osk with Vas and Stau. Because interactions with Vas and Stau are affected similarly, it is possible that all three mutations cause a general change in Osk protein conformation and render Osk unable to engage in proper interactions.

\section{Short Osk but not Long Osk recruits Vas in ovaries}

Short Osk can assemble pole plasm in the ovary, but Long Osk cannot (Markussen et al. 1995). Moreover, Short Osk, but not Long Osk, interacts efficiently with Vas in yeast. We speculated, therefore, that the different abilities of the two Osk isoforms in inducing pole plasm are attributable to their different competence in recruiting Vas. To this end, we constructed two transgenes, short osk-bcd and long osk-bcd, and generated transgenic flies expressing either Short Osk or Long Osk at the anterior of oocytes.

Short Osk expressed at the anterior causes formation of bicaudal embryos that develop pole cells at their anterior, demonstrating that this isoform induces a functional pole plasm at an ectopic site (data not shown). Indeed, Short Osk recruits Vas to the anterior, as revealed by staining the short osk-bcd embryos with antibodies directed against Vas (Fig. 5A,C). On the other hand, Long Osk produced at the anterior has no discernible effect, and the resulting embryos are indistinguishable from the wild type (data not shown). This strongly suggests that Long Osk has little or no pole-plasm-inducing activity. Probing the long osk-bcd embryos with anti-Vas antibodies fails to reveal Vas protein at the anterior (Fig. 5B,D). We observe that the amount of Long Osk present at the anterior of long osk-bcd embryos is similar to the amount of Short Osk at the anterior of short osk-bcd embryos (Fig. 5E-H). We exclude, therefore, the possibility that a quantitative difference between the two Osk isoforms accounts for the difference in recruitment of Vas to the anterior. Rather, we conclude that Short Osk interacts effectively with Vas, but Long Osk does not. Furthermore, the failure of Long Osk to interact with Vas suggests that the amino-terminal region of Long Osk, not present in Short Osk, has an inhibitory effect on the Vas-interaction domain of Osk. Stau is detected at the anterior of both long osk-bcd and short osk-bcd embryos (data not shown). However, the bcd 3'UTR present in the construct, in addition to endogenous bcd RNA, has been shown to recruit Stau to the anterior effectively (Ferrandon et al. 1994). Hence, it is difficult to evaluate possible differences in amounts of Stau in this context.

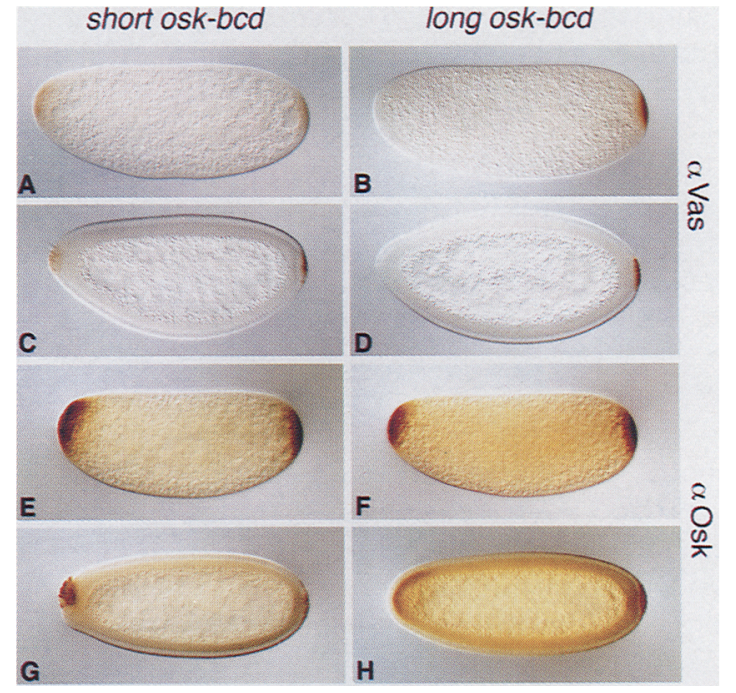

Figure 5. Short Osk, but not Long Osk, recruits Vas to the anterior pole of embryos. Embryos are oriented anterior pole at left, posterior pole at right. Embryos from females transgenic for short osk-bcd $(A, C, E, G)$ or long osk-bcd $(B, D, F, H)$ show normal posteriorly localized Vas $(A-D)$. Anteriorly localized Vas is detected in preblastoderm $(A)$ and blastoderm $(C)$ embryos from short osk-bcd females but not in embryos from long osk-bcd females $(B, D)$. Endogenous Osk is detected at the posterior pole of preblastoderm $(E, F)$ and blastoderm $(G, H)$ embryos, and transgenic Short Osk $(E, G)$ and Long Osk $(F, H)$ are detected at the anterior. The failure to recruit Vas to the anterior pole cannot be explained by insufficient levels of Long Osk, because the amounts of anterior Long Osk are similar to the amounts of anterior Short Osk.

Dynamic distribution of Stau, Osk, and Vas in ovaries and embryos

Analysis of Osk protein-binding activities suggests that Osk engages in at least two types of interactions, one with Vas and one with Stau. These interactions could take place within two distinct complexes, or within one that includes all three proteins. osk mRNA and Stau protein begin to localize to the posterior of the oocyte at stage 8 (Ephrussi et al. 1991; Kim-Ha et al. 1991; St Johnston et al. 1991). Shortly thereafter, the Osk proteins are detected at the posterior pole (Kim-Ha et al. 1995; Markussen et al. 1995). Vas protein is first observed at the posterior pole at stage 10a (Hay et al. 1990; Lasko and Ashburner 1990). All four proteins, Stau, Vas, and the two Osk isoforms, remain at the posterior through the end of oogenesis. In the embryo, Long Osk and Stau disappear from the posterior pole before the pole cell stage, whereas Short Osk and Vas remain at the posterior and are incorporated into the pole cells (Hay et al. 1990; St Johnston et al. 1991; Markussen et al. 1995).

To gain more insight into the different localization patterns, we examined the distribution of the different proteins relative to each other by confocal microscopy. We began by expressing a transgene consisting of Vas fused to GFP (Chalfie et al. 1994). GFP-Vas is detected in 
the same locations and with a similar granular distribution as endogenous Vas, within the egg chamber and the embryo (data not shown). Furthermore, the GFP-Vas transgene rescues abdominal and germ-line defects of vas (data not shown), demonstrating that GFP-Vas has vas activity. Thus, GFP-Vas serves as a marker for pole plasm, and presumably polar granules, relative to which the position of other proteins can be determined by antibody staining.

At the posterior pole of stage 10 oocytes, Osk and Stau show considerable overlap in their distributions with GFP-Vas (Fig. 6A-F). In early embryos, GFP-Vas is present in granular structures in a region near the posterior cortex. Osk is detected in the same subcortical region, and is concentrated in granular structures often coincident with the GFP-Vas positive spots (Fig. 6G-I). In contrast, Stau antibodies stain a thin crescent apposed closely to the posterior cortex of early embryos, revealing little overlap with GFP-Vas (Fig. 6J-L). Whereas Stau signal disappears from the posterior pole before the pole cell stage, Osk and Vas signals continue to overlap during pole cell formation (data not shown). This reveals that, in embryos, Osk colocalizes with GFP-Vas, but Stau does not. Hence, whereas Osk, Vas, and Stau colocalize in stage 10 oocytes, in early embryos, Stau shows a distinct localization pattern. Together with the interaction data, these results suggest that at least during one stage of oogenesis, all three proteins, Osk, Stau, and Vas, are present within one structural complex at the posterior pole. Later, however, complexes may exist that contain Osk and Vas, but not Stau.
Because Vas is a component of the polar granules, it is conceivable that the granular Osk and GFP-Vas signals near the posterior cortex of early embryos represent polar granules. In the electron microscope, we observe polar granules as dense structures located specifically at the posterior cortex of early embryos, in close proximity to mitochondria. To test whether Osk is a component of polar granules, we performed immunostaining on thin sections of early embryos, using an Osk-specific primary antibody. Gold particles are detected at high concentration on the polar granules, indicating that Osk is an integral component of the polar granules (Fig. 6M).

\section{Discussion}

Pole plasm assembly is initiated by recruitment of factors by Osk to the site where the Osk protein is localized. It has therefore been assumed that this process involves the interaction of Osk with known, and presumably additional unknown, pole plasm components. In this study, we have shown both in yeast and in vitro that Osk interacts directly with two pole plasm proteins, Vas and Stau, and that the in vitro interactions are affected by mutations in Osk that affect pole plasm assembly.

\section{Osk-Vas interaction essential for polar granule assembly}

Concurrent with the localization of Vas at the posterior pole, polar granules appear around stage 10 at the poste-

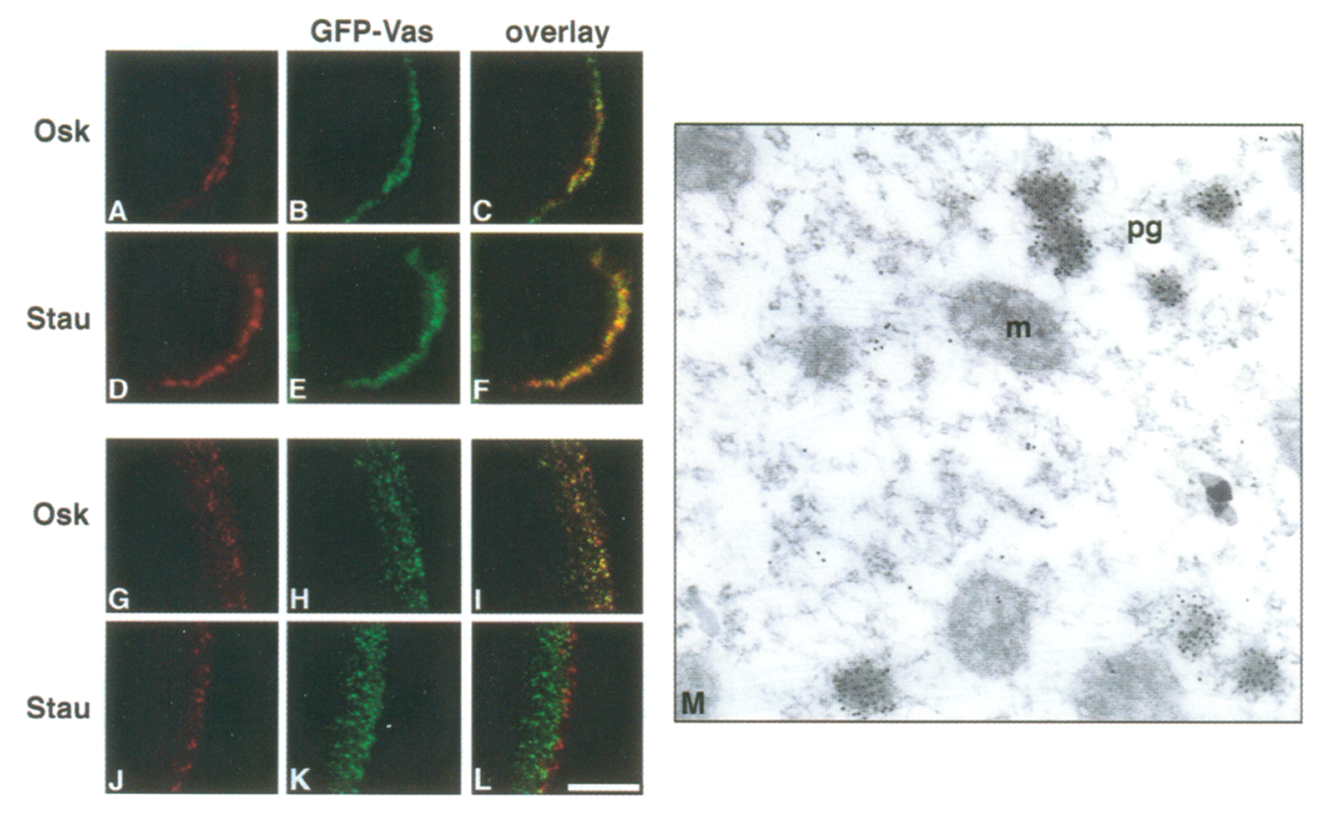

Figure 6. $(A-L)$ Osk and Stau colocalize with GFP-Vas in oocytes, whereas only Osk colocalizes with GFP-Vas in embryos. Double channel recordings of optical sections of stage 10 oocytes $(A-F)$ and early cleavage embryos $(G-L)$ show pole plasm staining with anti-Osk or anti-Stau antibodies in the presence of GFP-Vas. Red channel reveals Osk $(A, G)$ or Stau $(D, I)$, green channel reveals GFP-Vas $(B, E, H, K)$. Osk and GFP-Vas show strong overlap (yellow) in oocytes $(C)$ and embryos $(I)$, as revealed by overlay of channels. Stau and GFP-Vas show strong overlap in oocytes $(F)$ but little overlap in embryos $(L)$. Bar in $L$ indicates $10 \mu \mathrm{m}$. $(M)$ Osk is a component of polar granules. Gold particles visualizing Osk signals are highly concentrated on polar granules (pg) typically in close proximity to mitochondria $(\mathrm{m})$. 
rior of the oocyte (Hay et al. 1990). In accordance with its predicted function, Vas protein exhibits RNA-binding and helicase activities in vitro (Liang et al. 1994). The single missense mutations found in several vas alleles result in mutant Vas proteins whose RNA-binding and RNA helicase activities are defective in vitro. Nonetheless, these mutant proteins are localized properly at the posterior pole of the oocyte. Hence, the RNA-binding activity of Vas and localization of Vas to the posterior pole appear to be independent properties, suggesting that Vas localization occurs not through its association with localized RNAs, but rather through protein-protein interactions (Liang et al. 1994). Our study suggests that the Osk-Vas interaction mediates localization of Vas to the posterior pole. Based on the facts that mutant Osk proteins are defective in their interactions with Vas in vitro and that Osk and Vas colocalize to the polar granules, we propose that formation of an Osk-Vas complex represents an essential step in polar granule assembly.

In all examined osk alleles, Vas protein remains unlocalized (Hay et al. 1990; Lasko and Ashburner 1990). In these mutants, Vas is stable, yet fails to induce ectopic pole plasm. In this context, the simplest interpretation of our results would be that Osk acts as an anchor for Vas at the posterior pole. However, posterior localization of Vas is not strictly required for its activity. Indeed, Osk protein produced from a uniformly distributed source of osk mRNA in the oocyte causes ectopic activation of the posterior determinant (Kim-Ha et al. 1995). Thus, delocalized Osk, in conjunction with an unlocalized and abundant source of Vas present in the oocyte cytoplasm, can induce ectopic pole plasm. This indicates that the interaction of Vas with Osk, but not posterior localization of Vas, is the crucial factor in pole plasm formation.

\section{Polar granules and the regulation of Osk accumulation}

While inducing polar granule assembly, Osk protein accumulation is subject to regulation by pole plasm components. In alleles of vas or tud, the amount of Short Osk is significantly reduced compared with the wild type (Markussen et al. 1995; Rongo et al. 1995). Short Osk accumulation is also perturbed in the osk missense alleles that result in defective protein interactions in vitro (Markussen et al. 1995; W. Breitwieser, unpubl.). Whereas in the wild type, Short Osk is phosphorylated (F.-H. Markussen, unpubl.), this modification is not observed in these missense alleles. Because phosphorylation of Short Osk also fails in vas mutants, it is conceivable that a normal Osk-Vas interaction is required for modification of Short Osk.

Abundance of Short Osk protein could be regulated by modulation of its stability. Pole plasm is thought to protect certain RNAs from degradation (Ding et al. 1993). Similarly, pole plasm may protect proteins from degradation. The specific interaction of Short Osk with Vas, and the subsequent assembly of larger structures could ensure protection of Osk from degradation. Alternatively, the same proteins could influence the abundance of Short Osk by stimulating its efficient translation. Because Vas binds RNA and is active as an RNA helicase in vitro (Liang et al. 1994), Vas could well modulate translation of osk. The Osk-Vas protein interaction could mediate the recruitment of Vas to an RNP-complex containing osk RNA as a component. Because Vas, Tud, and in addition, Osk itself, are components of the polar granules (Hay et al. 1988a; Bardsley et al. 1993; this work), it is quite likely that functional polar granules are required for either stable accumulation or efficient translation of Short Osk.

\section{Possible role of the Osk-Stau interaction}

A complex mechanism is involved in the transport of osk mRNA into the oocyte and its localization and maintenance at the posterior pole (Ephrussi et al. 1991; Kim-Ha et al. 1991|. Because translation of osk mRNA occurs only once it is localized, we imagine that a key step in this process is the maintenance of osk mRNA at the posterior. Osk protein itself is involved in this maintenance function (Ephrussi et al. 1991; Kim-Ha et al. 1991). Indeed, although initially localized to the posterior pole, osk mRNA is released prematurely in osk nonsense mutants that fail to produce full-length Osk protein. Stau, required for both posterior localization and efficient translation (Kim-Ha et al. 1995) of osk, also fails to be maintained at the posterior of these alleles $/ \mathrm{St}$ Johnston et al. 1991).

The biological significance of the direct interaction between Osk and Stau is unclear. In the two-hybrid assay, both Long Osk and Short Osk interact with Stau, although Long Osk does so only weakly. In experiments where only the Long Osk isoform is expressed, the protein is abundant and stable at the posterior pole (Markussen et al. 19951. We assume, therefore, that osk mRNA and Stau protein are maintained at the posterior pole sufficiently to allow translation of Long Osk. Hence, Long Osk could support this maintenance function. In our experiments, the Osk-Stau interaction detected in vitro is diminished by missense mutations in the carboxy-terminal region of Osk. This does not seem to be the case in the ovaries of females expressing these osk missense alleles, in which Stau maintenance appears normal (St Johnston et al. 1991). It is possible that in the missense mutants, Stau localization could be affected, but not so severely as to be revealed by antibody staining. Alternatively, because Osk and Stau are likely to be components of a larger structure, additional stabilizing factors could compensate for a defective Osk protein.

Osk is a component of different complexes at different developmental stages

The observation that the distributions of Osk and Stau coincide in the oocyte, but are distinct in the embryo, suggests that Osk is a component of different complexes. Osk and Stau are both detected at the posterior pole of the oocyte at stage 9, whereas Vas is detected there at stage 10. A complex containing Osk and Stau could pro- 
vide a scaffold for osk transcript until structures containing Vas as a component are established. It is likely that polar granules are the manifestation of such structures. The presence of precursors of polar granules in oocytes at stage 9, before the genuine polar granules become apparent (at stage 10), has been reported (Mahowald 1962). Ultimately, immunoelectron microscopy will prove whether Osk and Stau colocalize to such precursor structures.

It is evident that synthesis of Osk protein is tightly regulated. Different mechanisms have been proposed as to how Osk itself is involved in this process (Webster et al. 1994; Rongo et al. 1995), but experimental support for these models has been lacking. Here we argue that the direct interactions in which Osk engages represent one aspect of osk autoregulation. The postulated function of the proteins constituting the polar granules is to store and translate mRNAs, such as those of the abdominal and germ-line determinants. It will be interesting to see whether, in addition to its protein-binding ability, Osk also binds RNA, and, for example, confers specificity for Vas helicase to certain RNAs. Alternatively, Osk could act solely by binding other proteins, inducing assembly of protein-RNA complexes.

\section{Materials and methods}

\section{Yeast two-hybrid assay}

The yeast two-hybrid assay was performed using the interaction trap as described (Gyuris et al. 1993). The yeast strain used was EGY48 (Mata his3 ura3-52 trp1 3lexA-op-leu2). Reporter plasmid pSH18-34ASpe (URA3 ${ }^{+}$8lexA-op-lacZ, obtained from $\mathrm{R}$. Brent, Massachusetts General Hospital and Harvard Medical School, Boston, MA) was linearized with ApaI and integrated into the URA3 locus. LexA-fusion proteins were expressed from plasmid pEG202 (Gyuris et al. 1993). For efficient import of Osk-fusions into the nucleus, plasmid $p L e x-N L S$ was constructed, containing the sequence for an SV40 large $T$ nuclear localization signal (encoding the amino acids CTPPKKKRKV; Silver et al. 1989) immediately downstream of LexA sequences. All osk sequences were subcloned from pBlue-osk containing the complete osk cDNA (Ephrussi et al. 1991). Osk amino acid sequence positions are relative to the first methionine used for translation, nucleic acid positions relative to the first initiation codon. pLex-NLS-Osk(1-606), expressing LexA-Long Osk, was made by inserting a 1922-bp EcoRI fragment of the osk cDNA into a pLex-NLS variant containing the bp 1-18 of the osk coding sequence. To make $p$ Lex-Osk(139-606), expressing LexAShort Osk, an osk cDNA fragment (bp 415-1940) was amplified by PCR and subcloned into $p L e x-N L S$. pLexA-Osk(7-468) was made by inserting an EcoRI-DraI fragment (bp 19-1404) into $p L e x-N L S$. pLexA-Osk(451-606) was made by deleting osk sequences $5^{\prime}$ of PstI (bp 1353) from pLex-Osk(1-606). pLexAOsk(290-606) was made by deleting all osk sequences $5^{\prime}$ of an EcoRV site (bp 867) from pLex-NLS-Osk(1-606). pLexA-Vas was made by cloning the complete vas coding sequence into $p E G 202$. All fusions with the transcription transactivator $\mathrm{B} 42$ were cloned into p/G4-5 (Gyuris et al. 1993). pB42-Vas was made by cloning the complete vas coding sequence into $p / G 4-5$. $p B 42-$ Vas $-C$ was made by deleting vas sequences $5^{\prime}$ of $E c o$ RI at position 1334 relative to the translation start codon. $p B 42-$ Vas- $N$ was made by inserting a PCR fragment of vas (bp 1-960) into $p / G 4$-5. $p B 42-S t a u-N$ and $p B 42-S t a u-C$ were constructed as insertions of two fragments of stau cDNA (bp 1-1704 and bp 1705-3085, relative to the stau translation intiation sitel. $p B 42$ $T u d$ fusions were cloned as insertions of fragments derived from tud cDNA clones $I O Z, 9 A 1$, and $3 Z S+L$ /Golumbeski et al. 1991 ) into $p / G 4-5$. PCR-cloning using Pfu polymerase (Stratagene) was verified by DNA sequencing. Plasmids $p R F H M 12$, $p L e x-M A X, p R F H M 7-3$ encoding LexA fused to DmCdc2, to human Max, and to the Ftz homeodomain, as well as plasmids p4-5-Cdi2, p4-5-Mxi1, encoding activation-tagged DmCdi2 and human Mxil, were obtained from R. Finley (Massachusetts General Hospital, Boston, MA) (Zervos et al. 1993; Finley et al. 1996). DNA-binding abilities of the different LexA-Osk fusion constructs were monitored in a repression assay using reporter plasmid $/$ K101 (Brent and Ptashne 1984). Yeast transformations and $\beta$-galactosidase assays were done using standard procedures (Ausubel et al. 1987-1993; Schiestl and Gietz 1989). For quantitative $\beta$-galactosidase assays, 4 independent yeast clones were tested and the average was determined.

\section{In vitro interactions}

Protein expression and purification: GST-Osk292 was made as an insertion of an osk cDNA encoding residues 292-606 into $p G E X-2 T K$ (Pharmacia). Mutations in GST-Osk292 were cloned by replacing wild-type restriction fragments with mutant fragments obtained by PCR from genomic DNA isolated from $o s k^{6 B 10}$, osk ${ }^{88}$, and $o s k^{150}$ homozygous female flies. Expression of GST and GST-Osk292 was induced in bacteria by adding IPTG to $0.6 \mathrm{~mm}$ for $3 \mathrm{hr}$ at room temperature. Cells were harvested and incubated in $50 \mathrm{~mm} \mathrm{Na}-\mathrm{PO}_{4} \mathrm{pH} 8.0,300 \mathrm{mM} \mathrm{NaCl}$, $2 \mathrm{~mm}$ DTT, $1 \mathrm{~mm}$ EDTA, $1 \mathrm{~mm}$ PMSF, 1\% Triton X-100, 200 $\mu \mathrm{g} / \mathrm{ml}$ Lysozyme (Sigma) for $15 \mathrm{~min}$ on ice and lysed by sonication. GST fusion proteins were purified using glutathione agarose beads (Sigma) and eluted from beads by adding $50 \mathrm{~mm}$ reduced glutathione (Sigma). Released protein was dialysed in 50 $\mathrm{mM} \mathrm{Na}-\mathrm{PO}_{4} \mathrm{pH} 8.0,300 \mathrm{~mm} \mathrm{NaCl}, 0.1 \%$ Triton X-100, $10 \%$ glycerol. Aliquots were kept frozen prior to use. Ovary extracts: for each binding reaction, 50 flies were dissected in Ringers solution. Ovaries were homogenized in extraction buffer containing $25 \mathrm{~mm}$ HEPES pH 7.5, $300 \mathrm{~mm} \mathrm{NaCl}, 1.5 \mathrm{mM} \mathrm{MgCl}_{2}, 0.2$ mM EDTA, $0.1 \%$ Triton X-100, $0.5 \mathrm{~mm} \mathrm{DTT}$, in the presence of protease inhibitors $(1 \mu \mathrm{g} / \mathrm{ml}$ leupeptin, $1.4 \mu \mathrm{g} / \mathrm{ml}$ pepstatin A, 5 $\mu \mathrm{g} / \mathrm{ml}$ aprotinin, $2 \mathrm{~mm}$ benzamidine, 0.5 mM PMSF, all Sigma), incubated for $30 \mathrm{~min}$ at $4^{\circ} \mathrm{C}$ and spun at $10,000 \mathrm{~g}$ twice for 15 min. Extracts were diluted 3 times with dilution buffer $125 \mathrm{~mm}$ HEPES pH 7.5, $2.5 \mathrm{~mm} \mathrm{MgCl}_{2}, 0.2 \mathrm{~mm}$ EDTA, $0.05 \%$ Triton $\mathrm{X}-100,0.5 \mathrm{~mm} \mathrm{DTT}$ ) containing protease inhibitors. In vitro translation: cDNAs encoding full length Vas, Stau, Stau-C (residues 569-1026), Stau-N (residues 1-568), and Nos were cloned into a transcription/translation plasmid modified from vector FPD13T (Glotzer et al. 1991). An Arbacia Cyclin B cDNA was expressed from $F P \Delta 13 T$. Proteins were made in vitro using TNT Coupled Reticulocyte Lysate Systems (Promega) in the presence of $\mathrm{T} 7$ polymerase and ${ }^{35} \mathrm{~S}$-methionine (Amersham). For binding assays using native Drosophila ovary extracts, 5 or $10 \mu \mathrm{g}$ of GST-Osk292 or GST were bound to glutathione agarose and incubated with extracts, rotating for $4 \mathrm{hr}$ at $4^{\circ} \mathrm{C}$. Beads were washed in $25 \mathrm{~mm}$ HEPES pH 7.5, $75 \mathrm{~mm} \mathrm{NaCl}, 2.5 \mathrm{mM} \mathrm{MgCl}_{2}$, $0.2 \mathrm{~mm}$ EDTA, $0.05 \%$ Triton X-100, 0.5 mM DTT, and protease inhibitors, at a concentration half of that mentioned above. Proteins bound to beads were eluted by boiling in $1 \times$ SDS-gel loading buffer and separated by SDS-PAGE. Samples were blotted on PVDF-membranes (Millipore) and probed with antibodies using ECL reagents (Amersham). For interactions with labeled in vitro translated proteins, $5 \mu \mathrm{l}$ aliquots of lysates were diluted in 50 
mM HEPES pH 7.5, $150 \mathrm{mM} \mathrm{NaCl}, 3 \mathrm{~mm} \mathrm{MgCl}_{2}, 0.1 \%$ Triton $\mathrm{X}-100,2 \mathrm{~mm}$ DTT, $0.5 \mathrm{~mm}$ PMSF, incubated with $10 \mu \mathrm{g}$ GSTfusion proteins for $3 \mathrm{hr}$ at $4^{\circ} \mathrm{C}$, then washed with the same buffer. Interactions were quantified using a PhosphorImager (Molecular Dynamics).

\section{Transgenic flies}

The short and long osk-bcd constructs are based on the oskM1L and oskM139L constructs, respectively (Markussen et al. 1995). oskM1L has a mutation in the first start codon, and produces only Short Osk protein. oskM139L has a mutation in the second start codon and produces only Long Osk protein. The respective start codon-mutations were cloned as 514-bp BamHI-BssHII fragments into osk-bcd3'UTR (Ephrussi and Lehmann 1992). Transgenic flies were generated by $\mathrm{P}$ element-transformation (Rubin and Spradling 1982) of a $w^{1118}$ stock using the CaSpeR vector (Pirrotta 1988). Embryos were collected from individual transformed lines and stained with anti-Osk and anti-Vas antibodies as described (Ephrussi and Lehmann 1992).

\section{Immunofluorescence and immunoelectron microscopy}

GFP coding sequence was subcloned from pTU65 (obtained from M. Chalfie, Columbia University, New York, NY) into pCaTubMatpolyA (Ferrandon 1994). The GFP sequence in the resulting plasmid $p C a T u b-G F P$ is in frame with the first 9 codons of the Drosophila a4-tubulin gene (Kalfayan and Wensink 1981). vas coding DNA was subcloned downstream of GFP sequences. Expression of GFP-Vas from the resulting plasmid $p C a T u b-G F P-V a s$ is therefore under control of $\alpha 4-$ tubulin promoter and $\alpha 1-$ tubulin 3 ' untranslated sequences (Kalfayan and Wensink 1982). Transgenic flies were generated as described above. Rescue activity for abdomen and germ-line by GFP-Vas was determined in vasPD/vasD1 trans-heterozygote female flies (Schüpbach and Wieschaus 1986; Lasko and Ashburner 1990). Ovary and embryo fixation and whole-mount antibody staining were done as described (Markussen et al. 1995). Primary antibodies were anti-Osk from rat (Ephrussi and Lehmann 1992), or anti-Stau from rabbit (St Johnston et al. 1991); secondary antibodies were rhodamine-conjugated anti-rat, or anti-rabbit from goat (Jackson Immunoresearch Laboratories). Images were obtained using the EMBL confocal laserbeam microscope. For immunoelectron microscopy, embryos (0-30 min) were fixed overnight in $8 \%$ paraformaldehyde, $250 \mathrm{~mm}$ HEPES $\mathrm{pH}$ 7.4 , and incubated three times for $10 \mathrm{~min}$ in $2.1 \mathrm{~m}$ sucrose, PBS, prior to freezing in liquid nitrogen. Processing for freeze substitution in Lowicryl HM-20 using a Reichert CS-Auto was done as described (van-Genderen 1991). Sections (100 nm) were incubated with a polyclonal anti-Osk antiserum (Ephrussi and Lehmann 1992) and visualized with colloidal gold (Tokuyasu 1980).

\section{Acknowledgments}

We thank Russ Finley and Roger Brent for strains, plasmids, and technical advice on the interaction trap and for LexA-antibodies; Martin Chalfie for the GFP plasmid; Michael Glotzer for the cyclin B plasmid; Paul Lasko and Yuh Nung Jan for Vas antibodies; Daniel St Johnston for Stau antibodies; Chris Rongo and Ruth Lehmann for sharing information on the osk ${ }^{6 B 10}$ mutant. We also thank Anne-Marie Michon for help in the embryo staining, Jola B. Glotzer for help in immuno-EM and advice on the confocal microscope, and Fiorenzo Peverali for technical advice. Finally, we thank Daniel St Johnston, Stephen Cohen, and
Giulio Superti-Furga for critical reading of the manuscript and discussions. W.B. was supported by a grant of the Boehringer Ingelheim Fonds. W.B. and F.-H.M. were supported by predoctoral fellowships from European Molecular Biology Laboratory.

The publication costs of this article were defrayed in part by payment of page charges. This article must therefore be hereby marked "advertisement" in accordance with 18 USC section 1734 solely to indicate this fact.

\section{References}

Ausubel, F.M., R. Brent, R.E. Kingston, D.D. Moore, J.G. Seidman, J.A. Smith, and K. Struhl, ed. 1987-1993. Current Protocols in Molecular Biology. Wiley \& Sons, New York, NY.

Bardsley, A., K. McDonald, and R.E. Boswell. 1993. Distribution of tudor protein in the Drosophila embryo suggests separation of functions based on sites of localization. Development 119: 207-219.

Brent, R. and M. Ptashne. 1984. A bacterial repressor protein or a yeast transcriptional terminator can block upstream activation in yeast. Nature 312: 612-615.

Chalfie, M., Y. Tu, G. Euskirchen, W.W. Ward, and D.C. Prasher. 1994. Green fluorescent protein as a marker for gene expression. Science 263: 802-805.

Ding, D., S. Parkhurst, S. Halsell, and H. Lipshitz. 1993. Dynamic Hsp83 RNA localization during Drosophila oogenesis and embryogenesis. Mol. Cell. Biol. 13: 3773-3781.

Eddy, E.M. 1975. Germ plasm and the differentiation of the germ cell line. Int. Rev. Cyt. 43: 220-280.

Ephrussi, A. and R. Lehmann. 1992. Induction of germ cell formation by oskar. Nature 358: 387-392.

Ephrussi, A., L.K. Dickinson, and R. Lehmann. 1991. oskar organizes the germ plasm and directs localization of the posterior determinant nanos. Cell 66: 37-50.

Ferrandon, D. 1994. "Mise en place de la polarité antero-postérieure de la Drosophile au cours de l' ovogenèse: Étude de la localisation de l'ARMm du morphogène bicoid au pôle antérieur de l'embryon." Ph.D. thesis, Université Louis Pasteur, Strasbourg, France.

Ferrandon, D., L. Elphick, C. Nüsslein-Volhard, and D. St Johnston. 1994. Staufen protein associates with the 3'UTR of bicoid mRNA to form particles that move in a microtubulc-dependent manner. Cell 79: 1221-1232.

Finley, R.L. Jr., B.J. Thomas, S.L. Zipursky, and R. Brent. 1996. Isolation of Drosophila cyclin D, a protein expressed in the morphogenetic furrow before entry into the $\mathrm{S}$ phase. Proc. Natl. Acad. Sci. 93: 3011-3015.

Frohnhöfer, H.G., R. Lehmann, and C. Nüsslein-Volhard. 1986. Manipulating the anteroposterior pattern of the Drosophila embryo. I. Embryol. Exp. Morph. 97 (Suppl.): 169-179.

Glotzer, M., A.W. Murray, and M.W. Kirschner. 1991. Cyclin is degraded by the ubiquitin pathway. Nature 349: 132-138.

Golumbeski, G.S., A. Bardsley, F. Tax, and R.E. Boswell. 1991. tudor, a posterior-group gene of Drosophila melanogaster, encodes a novel protein and an mRNA localized during midoogenesis. Genes \& Dev. 5: 2060-2070.

Gyuris, J., E.A. Golemis., H. Chertkov, and R. Brent. 1993. Cdil, a human $G 1$ and $S$ phase protein phosphatase that associates with Cdk2. Cell 75: 791-803.

Hay, B., L. Ackerman, S. Barbel, L.Y. Jan, and Y.N. Jan. 1988a. Identification of a component of Drosophila polar granules. Development 103: 625-640.

Hay, B., L.Y. Jan, and Y.N. Jan. 1988b. A protein component of Drosophila polar granules is encoded by vasa and has extensive sequence similarity to ATP-dependent helicases. Cell 
55: 577-587.

Hay, B., L.Y. Jan, and Y.N. Jan. 1990. Localization of vasa, a component of Drosophila polar granules, in maternal-effect mutants that alter embryonic anteroposterior polarity. $D e$ velopment 109: 425-433.

Heasman, J., J. Quarmby, and C.C. Wylie. 1984. The mitochondrial cloud of Xenopus oocytes: The source of germinal granule material. Dev. Biol. 105: 458-469.

Illmensee, K. and A.P. Mahowald. 1974. Transplantation of posterior pole plasm of Drosophila. Induction of germ cells at the anterior pole of the egg. Proc. Natl. Acad. Sci. 71: 10161020.

Kalfayan, L. and P.C. Wensink. 1981. $\alpha$-tubulin genes in Drosophila. Cell 24: 97-106.

-1982. Developmental regulation of Drosophila $\alpha$-tubulin genes. Cell 29: 91-98.

Kim-Ha, J., J.L. Smith, and P.M. Macdonald. 1991. oskar mRNA is localized to the posterior pole of the Drosophila oocyte. Cell 66: 23-35.

Kim-Ha, J., K. Kerr, and P.M. Macdonald. 1995. Translational regulation of oskar mRNA by Bruno, an ovarian RNA-binding protein, is essential. Cell 81: 1-20.

King, R.C. 1970 . Ovarian development in Drosophila melanogaster. Academic Press, New York, NY.

Lasko, P.F. and M. Ashburner. 1988. The product of the Drosophila gene vasa is very similar to eukaryotic initiation factor-4A. Nature 335: 611-617.

- 1990. Posterior localization of vasa protein correlates with, but is not sufficient for, pole cell development. Genes \& Dev. 4: 905-921.

Lehmann, R. 1995. Establishment of embryonic polarity during Drosophila oogenesis. Sem. Dev. Biol. 6: 25-38.

Lehmann, R. and C. Rongo. 1993. Germ plasm formation and germ cell differentiation. Sem. Dev. Biol. 4: 149-159.

Liang, L., W. Diehl-Jones, and P.F. Lasko. 1994. Localization of vasa protein to the Drosophila pole plasm is independent of its RNA-binding and helicase activities. Development 120: 1201-1211.

Mahowald, A.P. 1962. Fine structure of pole cells and polar granules in Drosophila melanogaster. J. Exp. Zool. 151: 201215.

Markussen, F.-H., A.-M. Michon, W. Breitwieser, and A. Ephrussi. 1995. Translational control of oskar generates Short Osk, the isoform that induces pole plasm assembly. Development 121: 3723-3732.

Pirotta, V. 1988. Vectors for P-mediated transformation in Drosophila. In Vectors: A survey of molecular cloning vectors and their uses (ed. R. L. Rodriguez and D.T. Denhart). Butterworths, Boston, MA.

Rongo, C., E.R. Gavis, and R. Lehmann. 1995. Localization of oskar RNA regulates oskar translation and requires Oskar protein. Development 121: 2737-2746.

Rubin, G.M. and A.C. Spradling. 1982. Genetic transformation of Drosophila using transposable element vectors. Science 218: 348-353.

Schiestl, R.H. and R.D. Gietz. 1989. High efficiency transformation of intact yeast cells using single stranded nucleic acids as carrier. Curr. Gen. 16: 339-346.

Schüpbach, T. and E. Wieschaus. 1986. Maternal-effect mutations altering the anterior-posterior pattern of the Drosophila embryo. Wilhelm Roux's Arch. Dev. Biol. 195: 302317.

Silver, P., I. Sadler, and M.A. Osborne. 1989. Yeast proteins that recognize nuclear localization sequences. I. Cell Biol. 109: 983-989.

St Johnston, D., and C. Nüsslein-Volhard. 1992. The origin of pattern and polarity in the Drosophila embryo. Cell 68: 201219.

St Johnston, D., D. Beuchle, and C. Nüsslein-Volhard. 1991. Staufen, a gene required to localize maternal RNAs in the Drosophila egg. Cell 66: 51-63.

St Johnston, D., N.H. Brown, J.G. Gall, and M. Jantsch. 1992. A conserved double-stranded RNA-binding domain. Proc. Natl. Acad. Sci. 89: 10979-10983.

Strome, S., and W.B. Wood. 1982. Immunofluorescence visualization of germ-line-specific cytoplasmic granules in embryos, larvae, and adults of Caenorhabditis elegans. Proc. Natl. Acad. Sci. 79: 1558-1562.

Strome, S. and W.B. Wood. 1983. Generation of asymmetry and segregation of germ-line granules in early C. elegans embryos. Cell 35: 15-25.

Tokuyasu, K.T. 1980. Immunochemistry on ultrathin frozen sections. Histochem. I. 12: 381-403.

van-Genderen, I.L. 1991. Subcellular localisation of Forssman glycolipid in epithelial MDCK cells by immuno-electronmicroscopy after freeze-substitution. I. Cell Biol. 115: 10091019.

Webster, P.J., J. Suen, and P.M. Macdonald. 1994. Drosophila virilis oskar transgenes direct body patterning but not pole cell formation or maintenance of mRNA in D. melanogaster. Development 120: 2027-2037.

Whitington, P.M. and K.E. Dixon. 1975. Quantitative studies of germ plasm and germ cells during early embryogenesis of Xenopus laevis. J. Embryol. Exp. Morph. 33: 57-74.

Zervos, A.S., J. Gyuris, and R. Brent. 1993. Mxil, a protein that specifically interacts with Max to bind Myc-Max recognition sites. Cell 72: 223-232. 


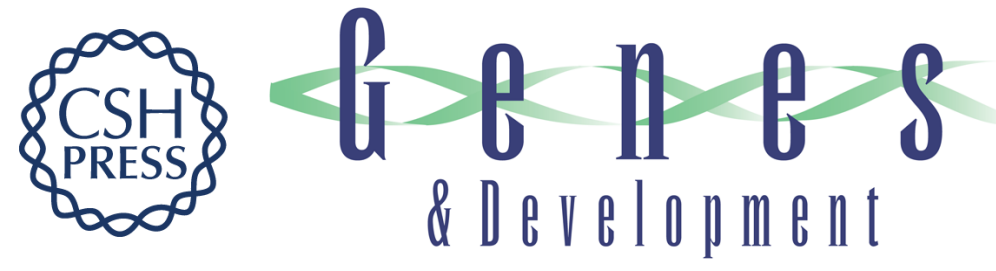

\section{Oskar protein interaction with Vasa represents an essential step in polar granule assembly.}

W Breitwieser, F H Markussen, H Horstmann, et al.

Genes Dev. 1996, 10:

Access the most recent version at doi:10.1101/gad.10.17.2179

References This article cites 44 articles, 18 of which can be accessed free at:

http://genesdev.cshlp.org/content/10/17/2179.full.html\#ref-list-1

License

Email Alerting

Service

Receive free email alerts when new articles cite this article - sign up in the box at the top right corner of the article or click here.

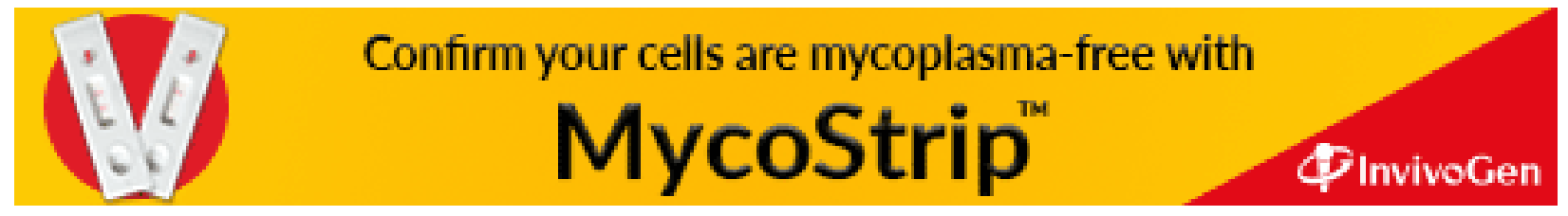

